\title{
An AI-based Incumbent Protection System for Collaborative Intelligent Radio Networks
}

\author{
Miguel Camelo*, Ruben Mennes*, Adnan Shahid ${ }^{\dagger}$, Jakob Struye*, Carlos Donato*, Irfan Jabandžić ${ }^{\dagger}$, \\ Spilios Giannoulis ${ }^{\dagger}$, Farouk Mahfoudhi*, Prasanthi Maddala ${ }^{\ddagger}$, Ivan Seskar ${ }^{\ddagger}$, Ingrid Moerman ${ }^{\dagger}$, and Steven Latré* \\ ${ }^{*}$ Department of Computer Science, University of Antwerp - imec, Antwerp, Belgium. \\ ${ }^{\dagger}$ imec - IDLab, Department of Information Technology at Ghent University, Ghent, Belgium. \\ ${ }_{\ddagger}$ WINLAB, Rutgers University, New Brunswick, NJ, USA. \\ Email: *[firstname.lastname]@uantwerpen.be, ${ }^{\dagger}\left[\right.$ firstname.lastname]@ugent.be, ${ }^{\ddagger}$ [prasanti, \\ seskar]@winlab.rutgers.edu,
}

\begin{abstract}
Traditionally, the radio spectrum has been allocated statically. However, this process has become obsolescence as most of the allocated spectrum is underutilized, and the part of the spectrum that is mainly used by the technologies that we use for daily communication is over-utilized. As a result, there is a shortage of available spectrum to deploy emerging technologies like 5G that require high demands on data. Several global efforts are addressing this problem, i.e., the Citizens Broadband Radio Service (CBRS) and Licensed Shared Access (LSA) bands, to increase the spectrum reuse by providing multi-tiers spectrum sharing frameworks in the re-allocated radio spectrum. However, these approaches suffer from two main problems. First, this is a slow process that may take years before authorities can reassign the spectrum to new uses. Second, they do not scale fast since it requires a centralized infrastructure to protect the legacy technology and coordinate and grant access to the shared spectrum. As a solution, the Spectrum Collaboration Challenge (SC2) challenge has shown that Collaborative Intelligent Radio Network (CIRN), i.e., Artificial Intelligence (AI)based autonomous wireless radio technologies that collaborate, can share and reuse spectrum efficiently without any coordination and with the guarantee of incumbent protection. In this paper, we present the architectural design and the experimental validation of an incumbent protection system for the next generation of spectrum sharing frameworks. The proposed system is a twostep AI-based algorithm that recognizes, learns, and proactively predicts the transmission pattern of the incumbent in near real-time, less than $300 \mathrm{~ms}$ to perform a prediction, with an accuracy above $95 \%$ to correctly predict where the incumbent is transmitting in the future. The proposed algorithm was validated in Colosseum, the RF channel emulator built for the SC2 competition, using up to two incumbents simultaneously, which have different transmission patterns, and sharing spectrum with up to 5 additional networks.
\end{abstract}

Index Terms-Spectrum sharing techniques, Incumbent protection, Cognitive Radios, Artificial Intelligence, Radio Spectrum Management, Inter-Network Collaboration, Collaborative Intelligent Radio Networks, 5G.

\section{INTRODUCTION}

New wireless technologies like $5 \mathrm{G}$ require more available radio spectrum to support new applications with high demands on data. However, their deployment is being limited due to a shortage of the available spectrum [1], [2]. One of the main reasons of this shortage is the obsolescence of the

Manuscript received December 1, 2012; revised August 26, 2015. Corresponding author: M. Shell (email: http://www.michaelshell.org/contact.html). traditional static frequency plan that is based on proving access to single usage, e.g., ISM unlicensed band, or single user, e.g., mobile operator in a licensed bands, with granted rights of exclusive use of a specific portion of the spectrum in a given geographic location. As a result, most of the allocated spectrum is underutilized, as today radio systems do not transmit all the time or everywhere and no one is allowed to take advantages on it, and the part of the spectrum that is mainly used by the technologies that we use for the daily communication is over-utilized.

This exclusive-usage spectrum allocation model is being addressed by several global efforts to make additional spectrum available for broadband data and to increase the spectrum reuse [3], [4]. The Citizens Broadband Radio Service (CBRS) band [5], $3550 \mathrm{MHz}$ to $3700 \mathrm{MHz}$ in the United States, and the Licensed Shared Access (LSA) band [6], $2300 \mathrm{MHz}$ to $2400 \mathrm{MHz}$ in Europe, are initiatives that provide multi-tiers spectrum sharing frameworks in re-allocated radio spectrum, which have been mainly used for military purposes (e.g., radio-location and satellite communication). Taking as an example the CBRS, it offers three-tiered access to federal and non-federal users via an automated frequency coordinator, known as a Spectrum Access System (SAS). The three tiers of authorization in this band are (from highest to lowest priority): the Incumbent Access, Priority Access, and General Authorized Access. In this scheme, once a higher priority user is transmitting, the lower ones must vacate the spectrum to avoid interference.

Although these multi-tier approaches are an initial step to mitigate the spectrum scarcity via spectrum sharing efficiently, it suffers from two main problems. The first problem is that the spectrum allocations authorities are not able to keep up with the growing demand; it takes years before authorities can re-assign the spectrum to new uses. The second problem is that this approach does not scale fast since it requires a centralized infrastructure to coordinate and grant access to the shared spectrum. Note that there are already spectrum sharing technologies, e.g., the WiFi Dynamic Frequency Selection (DFS) model, which implements a sense and avoid the principle of Dynamic Spectrum Access (DSA), that do not require centralized infrastructure to protect the incumbents. However, it lacks methods to avoid interference and provide 
incentives to make the protection of higher-tier users effective.

As a solution, the Spectrum Collaboration Challenge (SC2) challenge, a three-year competition organized by Defense Advanced Research Projects Agency (DARPA), has shown that Collaborative Intelligent Radio Network (CIRN), i.e., Artificial Intelligence (AI)-based autonomous wireless radio technologies that exchange explicit information to solve joint problems via collaboration, can share and reuse spectrum efficiently without any coordination and with the guarantee of incumbent protection [7]. This is a step beyond modern Cognitive Radio (CR) networks as they are limited to obtain and use local information via spectrum sensing.

In this paper, we present the architectural design and the experimental validation of an incumbent protection system for the next generation of spectrum sharing frameworks. The contributions of this paper are three-fold. Firstly, we design an architecture for an incumbent protection system that does not require any central infrastructure to control and grant access to shared spectrum based on the concept of CIRN. Secondly, we design and implement a two-step AI-based algorithm that, in combination with collaborative information, can recognize, learn, and proactively predict the periodic transmission pattern of an incumbent in near real-time and with an accuracy above 95\% can predict future incumbent transmissions in all the nodes. Finally, we validate our approach in Colosseum [8], the world's largest RF channel emulator built for the SC2 competition, using up to two incumbents simultaneously, which have different transmission patterns and sharing spectrum with up to 5 additional networks, each one composed by up to 10 nodes.

The rest of this paper is structured as follows. Section III introduces the spectrum sharing based on the concept of CIRN. The proposed two-step AI-based algorithm for incumbent protection is presented in Section III Implementation details and the integration in our collaborative intelligent radio technology is described in Section IV] The experimental results and performance evaluations are provided in Section V. Conclusions and future work are presented in Section VI

\section{TWO-TIER MODEL FRAMEWORK FOR CIRN}

The multi-tier incumbent protection model which our system is based on has the following properties:

1) It has two tiers, the protected incumbent and the other users.

2) Other users can use any spectrum voxel, where a voxel is defined in time, frequency, location, and power that is not used by the incumbent inside the shared spectrum band.

3) The users have no expectations of any interference protection.

4) There is an interconnection network for collaboration where the incumbent reports spectrum power measurements, which determines if non-incumbent RF transmissions are causing interference to it, and its frequency operation details (central frequency and bandwidth)

5) Radio networks are connected to the collaboration network where they receive incumbent reports.

6) Causing interference with the protected incumbent is penalized.

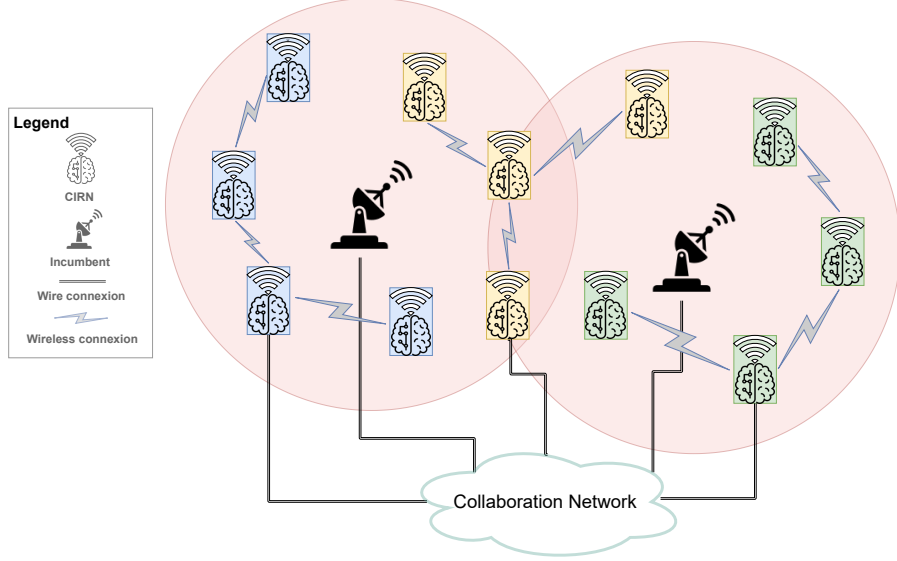

Fig. 1: Two-tier model framework for incumbent protection using CIRN

Notice that while properties 1-3 are shared with the DFS model [4], the properties 4-5 are novel and are used as a mechanism to incentivize and enforce the protection of the incumbent, and property 6 is shared with CBRS. Taking as an example the CBRS model, if the radios interfere with the incumbent, then the radios transmitting simultaneously in its coverage are, in a way penalized, by shutting them down.

This model is simpler and scales better, in the number of users/incumbents, than the CBRS and LSA frameworks since it does not require centralized authority controlling and granting access to the shared spectrum. However, it also implies that the incumbents require spectrum sensing capabilities to collaborate and share information about their spectrum power measurements, The radios also need to be intelligent, fully-autonomous, and collaborative to create Collaborative Intelligent Radio Networks (CIRNs).

Intelligent and fully autonomous radios will be able to dynamically learn and apply the best policy that determines their allocation of the spectrum, e.g., based on a given objective such as fairness, performance, etc., while simultaneously protecting the incumbents and minimizing the interference with other technologies that share the spectrum. However, cognitive capabilities in radios only exploit local views of the spectrum, which is not enough to protect the incumbent. Here is where collaboration plays a key role. Collaboration among networks and incumbents helps reduce the uncertainty that faces the autonomous radios making decisions based on their local view of the spectrum by enhancing it with global information from the environment. Figure 1 shows an overview of the two-tier framework for incumbent protection using CIRN.

During the SC2 competition, our team designed, implemented, and tested a CIRN called SCATTER [9]. A brief introduction to the architectural design of the SCATTER radios is presented below. It is composed of 6 fundamentals blocks: Physical (PHY) layer, Medium Access Control (MAC) layer, and User Data Management (UDM) layer, which are part of the data plane, the Intelligent Control and Decision Engine (ICDE), which is part of the control plane, the Radio Frequency Monitor (RF-MON), and the Collaborative Interface 
(CI). Figure 2 provides a simplified view of the SCATTER architecture and the sub-modules of the ICDE, which contains the building blocks for our incumbent protection system.

PHY: This layer is implemented as a Software Defined Radio (SDR) with features such as Orthogonal FrequencyDivision Multiplexing (OFDM) waveform, bursty transmission, dual-concurrent physical layers, Field-Programmable Gate Array (FPGA)-based filtered transmission, out-of-band full-duplex operations, and layer configuration based on timed commands [10].

MAC: This layer is based on an enhanced Multiple Frequencies Time Division Multiple Access (MF-TDMA) scheme that exploits the features provided by the PHY layer. Some of its capabilities are the distributed slot-allocation protocol, slot allocation with Quality of Service (QoS) support, and robust mechanisms against failures in slot allocation/removal procedures.

UDM: This layer monitors the incoming traffic from the user/application space in run-time and reports it to the control layer. Also, it provides the capabilities of reshaping bursty traffic to Constant Bit Rate (CBR)-like flow.

Intelligent Control and Decision Engine (ICDE): This module controls and optimizes the performance of the radio by combining rule-based and AI-based algorithms. The primary source of information is the RF-MON, e.g., spectrum samples, MAC, e.g., slot allocation status and link stats, the UDM, i.e., traffic flow information, and the CI, e.g., reports from the protected incumbent. After combining and consuming these data sources, the ICDE module intelligently and dynamically adapts the radio parameters at different layers to improve performance, increase spectrum efficiency, and collaborate with other networks to increase the spectrum sharing.

$\boldsymbol{R F - M O N}$ : This FPGA-based module provides the local view of the spectrum to the ICDE via spectrum sensing measurements.

CI: This module is the connection point between the SCATTER CIRN, the protected incumbents, and other CIRNs via a well-defined collaboration protocol or language. In general, it reports and receives messages from other networks/radios such as location, the actual and predicted spectrum voxel usage, performance metrics, among others, and the spectrum power measurement reports from the protected incumbents.

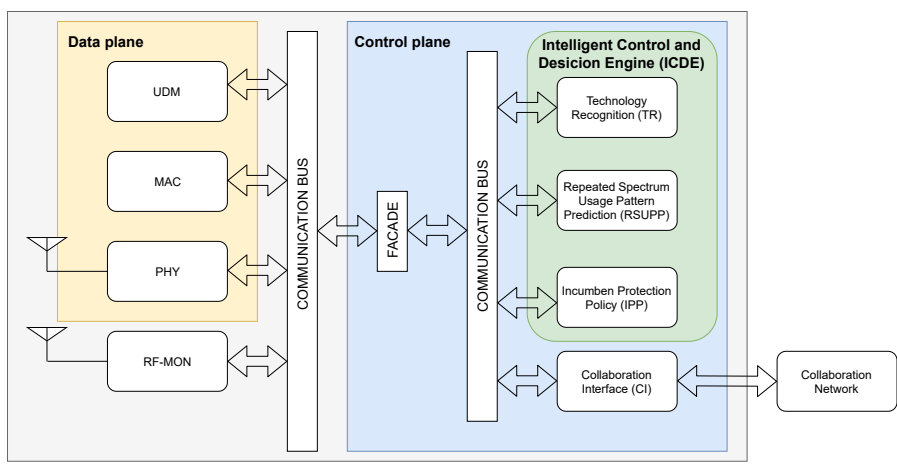

Fig. 2: SCATTER architecture overview and the ICDE components for incumbent protection
In the next section, we explain the functionalities of the internal modules of the ICDE and how they are orchestrated to solve the problem of protecting the incumbent with CIRN.

\section{INCUMBENT PROTECTION IN SCATTER}

Although the DFS scheme is simpler and easier to deploy than CBRS and LSA, it lacks 1) a mechanism to exploit the voxels that are not used by the incumbent and, if it does not work correctly, 2) a mechanism to incentive and enforce the incumbent protection. To overcome these limitations, the twotier sharing spectrum scheme with CIRNs must require that the Collaborative Intelligent Radio (CIR) can:

- Share what spectrum remains when the protected incumbent is active, and a sub-band is available.

- Dynamically exploits periods when the incumbent is pointing away from their radios.

- Use collaborative information to minimize the probability of harming the protective incumbent.

Translating it to radio capabilities, CIRs need not only to detect and identify radio technologies per spectrum voxel but to select the right policy to execute according to the identification and being pro-active to maximize the spectrum usage efficiency as reacting too late may impact your performance by reducing the transmission opportunities. To support it, SCATTER radios implement two AI-based modules inside their ICDE that provide identification and spectrum usage pattern prediction.

Technology Recognition (TR): This module implements a Convolutional Neural Network (CNN) that recognizes spectrum signatures of different radios technologies and idle (noise) separately [11]. As input, it consumes the continuous stream of spectrum data collected by the RF-MON block and outputs if a given technology is present in a two-dimensional spectrum voxel, only in time and frequency.

Repeated Spectrum Usage Pattern Prediction (RSUPP): Under the assumption that some technologies, like radars, uses the spectrum following a fixed time-frequency pattern, this module implements a Machine Learning (ML)-based algorithm that learns and predicts their patterns in real-time.

At this point, it is almost evident how these modules can be used to protect the incumbent. In the ideal case, if the active incumbent is in the vicinity of a given radio, then the radio's TR module should detect it. By filtering this information from other radio signatures, a new view of the $2 \mathrm{D}$ spectrum voxel is created: voxels where the incumbent is detected and voxels with no incumbents. This new view is forwarded to the RSUPP module, and, as the protected incumbent has a fixed and repeated transmission pattern, it should learn and predict the future usage of the spectrum by the incumbent. Note that this two-step procedure is required to support real-time decision making since applying online learning algorithms, e.g., Reinforcement Learning (RL), is not practical as using raw spectrum data introduces a large state-action space and long training time [12].

But, what if the TR is not able to recognize the incumbent due to other users are interfering with it? An example is when a CIRN starts accessing the spectrum for the first time, 


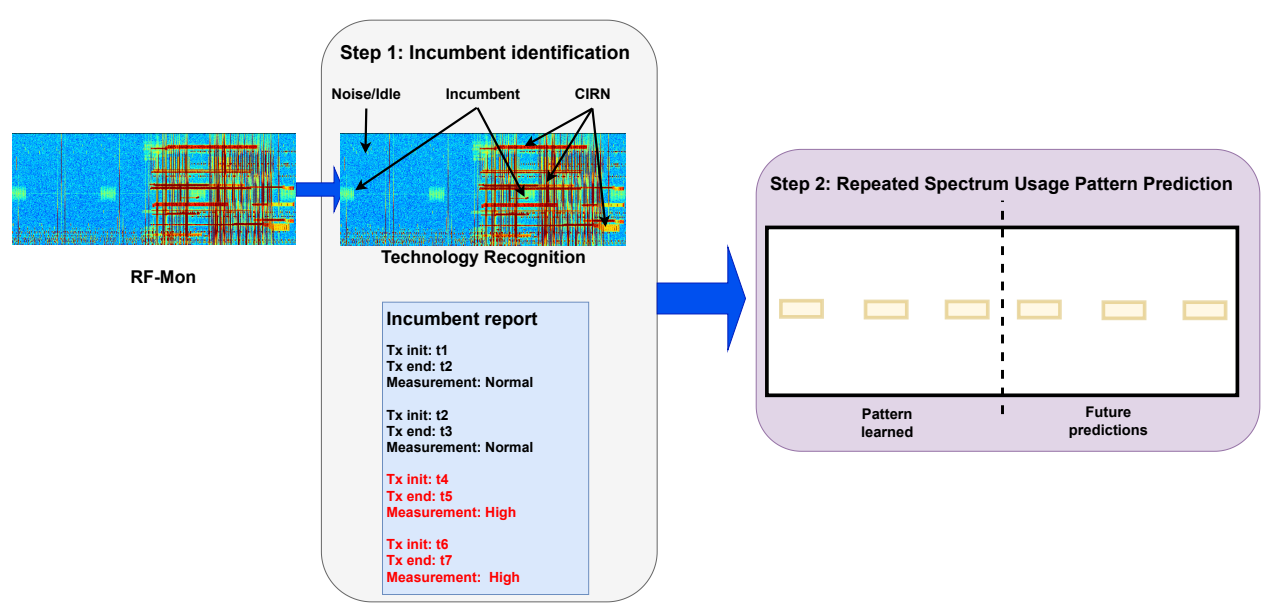

Fig. 3: Proposed two-step AI-based algorithm for incumbent protection

but no knowledge about the presence of the incumbent nor its pattern has been learned. In this case, the probability of recognizing it by the TR module drops drastically. It is at this point where the collaborative information plays a fundamental role: the spectrum power measurements, which are shared by the incumbent, reduce the uncertainty about the incumbent transmissions when the TR module is blind. The information provided by these measures guarantees that in the case of TR blindness due to the interference of others CIRNs with the incumbent, the RSUPP can use this information as ground truth of the incumbent existence. It also can work the other way around. If the incumbent is reporting interference, but the TR of a given radio has high confidence that the incumbent is not detected, then it can share the full spectrum with other CIRNs since it is not causing such interference. Figure 3 shows the general idea of the proposed two-step algorithm.

Incumbent Protection Policy (IPP): Depending on the technologies detected, a CIRNs may apply different policies while accessing the spectrum. As we focus on the incumbent, the application of the incumbent protection policy by the CIRs is guaranteed by the ICDE, given its autonomous nature to make decisions. Given that, once the RSUPP has learned the incumbent pattern, it forwards this information to this module and triggers the required changes on PHY/MAC to ensure the protection of the incumbent. The application of the policy results in changes in the transmission power and transmission bands, on the scheduling for future transmissions based on the timing of the learned patterns, among others.

Notice that this framework would allow an additional enforcement mechanism to guarantee protection. In this spectrum sharing framework, spectrum power measurements, cocerning a threshold limit on maximum transmission power to avoid harming the incumbent, can be used as an incentive (or punishment) to improve its protection accuracy. In the next section, we describe how the SCATTER radios implement the AI-based incumbent protection system, how the policy is defined based on the capabilities of the SCATTER PHY and MAC layers, and how the SC2 challenge implements the incentive mechanism to enforce the incumbent protection.

\section{IMPLEMENTATION}

Each SCATTER CIRN implements the two-step AI-based incumbent protection as follows. The RF-MON and TR follow the same implementation as the one described in [11] but with the following differences. Firstly, instead of using raw IQ samples, TR uses 32-averaged 512-point Fast Fourier Transform (FFT) samples at a sample rate of 23.04 Msps (or 46.08 Msps if the available bandwidth is $>20 \mathrm{MHz}$ ). These changes are translated into approximately $32 \mathrm{x}$ lesser examples to process in TR, in comparison to the raw In-phase and Quadrature (IQ) sample version. Although it comes at the cost of losing frequency resolution to detect the incumbent, i.e., the side lobes are almost not recognizable, we compensate it by using the frequency operation details shared by the incumbent via collaboration. Secondly, a module between RF-MON and TR, called AI Model Feeder, was implemented to link RF-MON samples to frames of our MAC. It provides a one-to-one map between our transmitting and receiving scheduling and the output of the TR module. Thirdly, we added the new incumbent radio signature class, and we grouped the other CIRNs signatures under the same class as there is no need for individual identification of them. Finally, our Deep Learning (DL) model was a CNN trained in a supervised way, and its input was fixed to 32 floating points, representing 32 FFT samples, as every channel in our MF-TDMA scheme was always a multiple of 16 or 32 FFT samples. This per-channel classification reduces the amount of resources required by the $\mathrm{CNN}$ model. Figure 4 shows the $\mathrm{CNN}$ architecture used for the SC2 challenge during the third year.

As the optimized data acquisition process provided by RF-MON is aligned with our MF-TDMA scheduling, via the AI model feeder module, we perform an additional step to compact the information before it is sent to the RSUPP. In this scenario, each SCATTER time-frequency voxel is composed of 35 32-FFT samples. It means there are 35 predictions about what technology is identifying in a given SCATTER timefrequency voxel. To maximize the protection of the incumbent and minimize the number of false-positives identifications, we define that the incumbent is detected in a given SCATTER time-frequency voxel if $\geq 15 \%$ of the samples are classified 


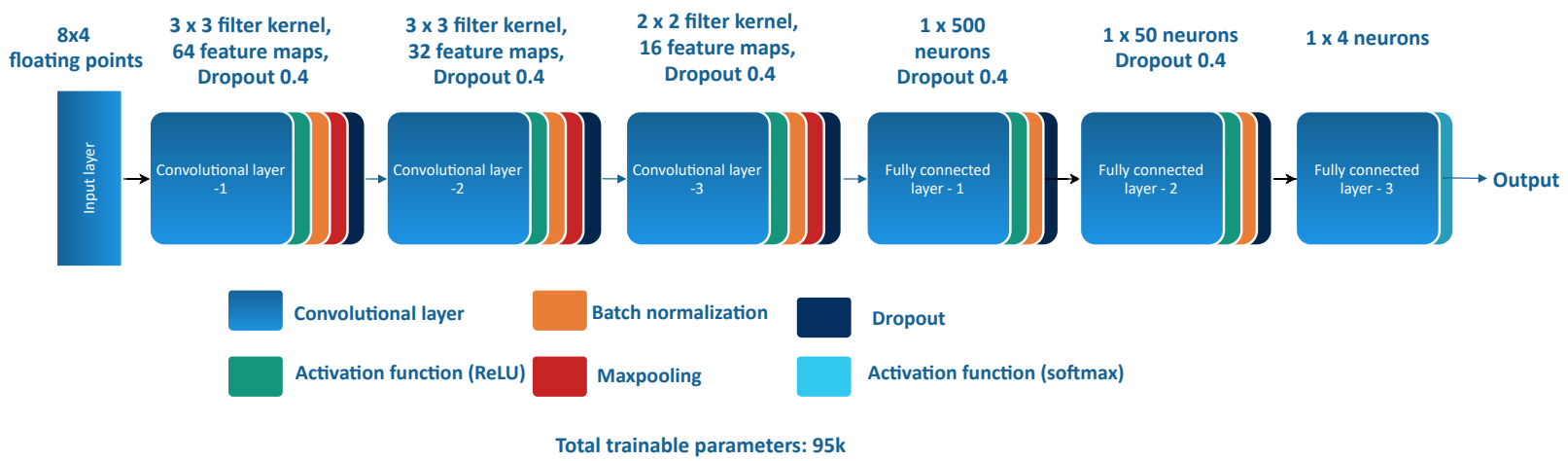

Fig. 4: CNN model used for TR. It only requires 95k parameters, which is translated in short training time and fast prediction in run time.

as incumbent with and accuracy $\leq 99 \%$. As a result, the implemented TR module provides a radio signal identification system that is aligned to our MF-TDMA scheme, highly accurate, and operates in real-time.

TR outputs a 2D Boolean grid, which aligns with our MF-TDMA scheduling, where one means the incumbent is detected in a given time-frequency voxel, zero otherwise. This output is received by the RSUPP, together with the reports from the incumbent. As the incumbent reports from the incumbent are timestamped, the RSUPP frames them with our MF-TDMA scheduling, creating a 2D grid similar to the one provided by TR. Then it combines the incumbent reports with the received from TR via an OR operation. The resulting grid is processed per channel. Notice that as the incumbent reports the central frequency and bandwidth, we reduce our computational requirements by processing only the channels that may overlap the incumbent (the incumbent channels). For each incumbent channel, the RSUPP creates a Boolean string on which the prediction will be executed. The last string characters, which are selected using the time frame and the average time of the incumbent pattern, are used as a prefix. This prefix is used to search in the buffered string that contains a fixed number of previous strings. This buffered string is used to create a probability tree that represents all possible incumbents states after the specified prefix. An example showing three possible trees with three different prefixes are shown in 5. In this implementation, we set the buffer size to 300 , the depth of the tree search to 100 , and the pattern to find will have a length between 20 and 100 .

This process is done for all the prefixes starting from the end string (reading in backward). Notice that by increasing the prefix length, we reduce the number of branches in the probability tree. The algorithm combines the different trees, and if the first $n$ children are reachable with a probability higher than a given threshold following the greedy path, the algorithm determines that it has found a pattern candidate. If the same pattern is detected a few times in a row, the pattern is used to avoid the incumbent on a given channel. Once a pattern is found, we fix and use it until we detect changes on it. This is very important to support changes over time or recovery after learning an incomplete or corrupted pattern. Although the length of the pattern could vary, we set a maximum size

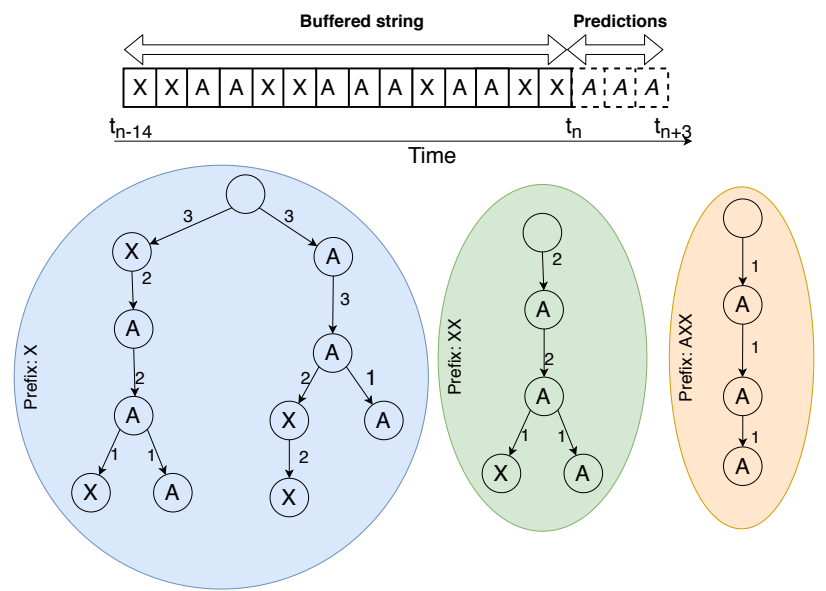

Fig. 5: Example of creating a probability tree based on current incumbent detection buffer. Were A represent Incumbent is present and $\mathrm{X}$ the absence of an incumbent during that specific time slot.

since searching for long patterns may imply that there is no a pattern and is computationally costly.

\section{EXPERIMENTAL VALIDATION}

The SC2 challenge provided a scenario to test the CIRNs capabilities to coexist and protect the incumbent. In this scenario, CIRNs were challenged not to harm a Terminal Doppler Weather Radar (TDWR) system, denominated as the Active Incumbent, while sharing and exploiting the spectrum voxels not used by the incumbent. This scenario had a duration of 330 seconds, where all the CIRNs were sharing $10 \mathrm{MHz}$ of bandwidth. The scenario was composed of up to five CIRNs, where one node per network, the gateway, was connected to the collaboration network.

The incumbent to-be protected shares the following information via an implemented collaboration protocol called CIRN Interaction Language (CIL) [13]: its current SignalTo-Interference-Plus-Noise Ratio (SINR); a threshold, in dB, that sets the minimum value on which other transmissions will start harming the incumbent; a violation flag is activated when the threshold is breached; and the incumbent center 

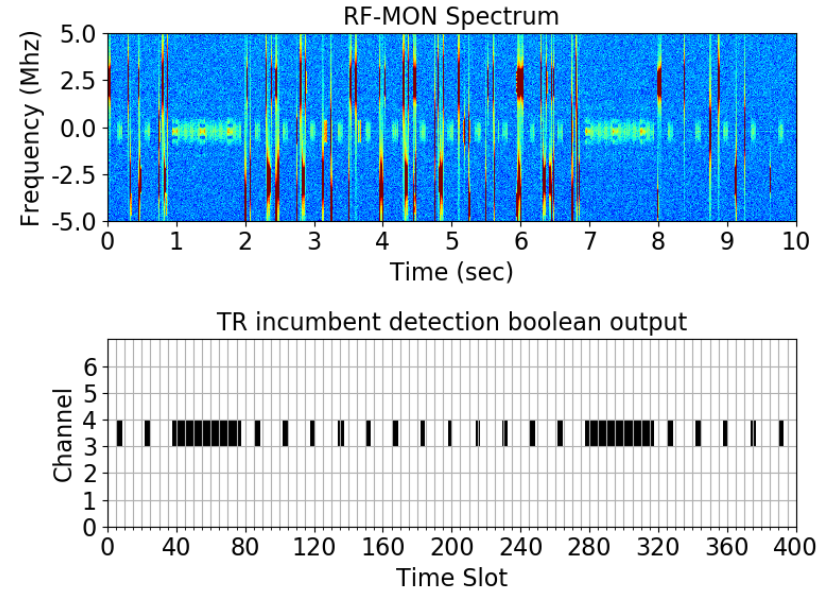
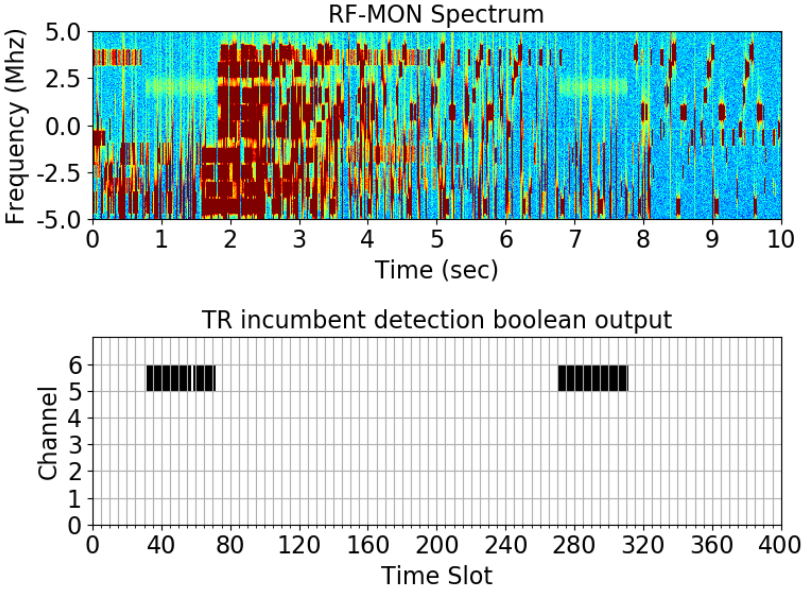

Fig. 6: CIRNs collaborating and protecting the Incumbent. a) SCATTER CIRN protecting two incumbents and b) Multiple CIRNs protecting an incumbent. Spectrum received from RF-MON (top) and the incumbent detection output from TR (bottom) with respect to the SCATTER MF-TDMA scheduling (1.4 MHz channels, 25ms per time slot)

frequency and bandwidth. This information was shared at a rate of $10 \mathrm{~Hz}$. CIRNs follow a simple procedure to access the network. Once the scenario started, CIRNs and the protected incumbent(s) register to a Collaboration server. Then, peerto-peer communication is established among CIRNs to begin collaborating. Traffic that CIRNs have to route among their nodes is rewarded with points if their requirements of QoS hold for a determined period.

The competition was designed to award the CIRN with the most scored points. However, CIRNs were responsible for computing their score and getting the score of other CIRNs via the collaboration network. This scoring system has two main properties: 1) if any CIRN is scoring points below a threshold, then all the CIRNs will receive the same score, and, 2 ) if the incumbent reports that it is harmed in a period, then the CIRNs receives no points for that period. While 1) was used to promote collaboration among CIRNs, motivating them to give spectrum to the weakest team, 2) is used to enforce and incentivize the CIRNs to protect the incumbent.

The scenario had three difficulty levels and two incumbent bandwidths. The three levels were the easy, where the incumbent follows a slow transmission pattern; the medium, where the incumbent transmits shorter and faster than the easy one; and the hard, where two incumbents are transmitting in the same band, one with the easy and the other with the fast pattern. For each difficulty, the incumbent was able to either use the full available bandwidth, therefore no other CIRNs can transmit at the same time, or half of the available bandwidth, then CIRN can transmit in the other half of the spectrum. In this section, we present two of the executions of this scenario were SCATTER was involved, as a single CIRN, and collaborating with multiple CIRNs. For visualization purposes, the relative transmission power of the incumbents received by RF-MON was modified (increased) to allow its visualization.

\section{A. SCATTER protecting the incumbent alone}

In this experiment, SCATTER CIRN was deployed alone to protect two incumbents transmitting in the same channel (hard level scenario) and using the full bandwidth. Figure 6 a) shows the spectrum view provided by RF-MON and the Boolean 2D grid that the TR module forwards to the RSUPP module. Note that although the incumbents were using the whole bandwidth, only its middle lobe has enough power to be recognized. As we mentioned before, this is a side effect of the average applied to the FFT samples by RF-MON to reduce the amount of data to be processed. However, the information shared by the incumbents provides the missed information in their reports (frequency operation data). Nevertheless, it does not impact the capabilities of TR to recognize the different incumbents correctly. SCATTER CIRs were able to learn the incumbent patterns and schedule their transmissions between the transmissions of the incumbents. In this experiment, $98 \%$ of the future transmissions of the incumbent were correct, and most of the mistakes were during the initial seconds of the scenario where the algorithm was in the learning phase.

\section{B. SCATTER and multiple CIRN protecting the incumbent}

In this experiment, three CIRNs were deployed, including SCATTER CIRN, to protect one incumbent in the easy level scenario and using half of the available spectrum. Figure $6 \mathrm{~b}$ ) shows how multiple CIRNs were able to protect the incumbent while maximizing the use of the spectrum. Depending on the traffic demands, CIRNs try to use the available spectrum left by the incumbent efficiently. For example, between second 0 and 4, high traffic demand is being managed by the CIRNs. However, no CIRNs scheduled transmissions in the $5 \mathrm{Mhz}$ band (upper band) while the incumbent was transmitting. Note that between the time-slots 50 and 60, there was one part of the incumbent that was not detected due to a side-band transmission of a CIR, which results in TR could not identify the incumbent there. However, this gap was well predicted by 
the RSUPP as an incumbent transmission. In general, 95\% of the future transmission of the incumbent were predicted correctly. This small drop of accuracy is expected since coexisting with other networks introduces a longer learning time.

\section{Execution time performance}

On average, the two-step algorithm took less than 300ms. TR used $200 \mathrm{~ms}$ to format the data received from the AI Model Feeder, i.e., transforming raw bytes into python arrays, $100 \mathrm{~m}$ for performing the TR task, and $50 \mathrm{~ms}$ to predict the transmission pattern of the incumbent for the next SCATTER frame. As the RSUPP is implemented in c++ , it can efficiently process the received raw bytes. Finally, learning a pattern varies, on average, from 5 seconds, in solo scenarios, up to 30 seconds, in coexistence with other CIRNs.

\section{CONCLUSIONS AND FUTURE WORK}

We presented the architectural design and the experimental validation of an incumbent protection system for the next generation of spectrum sharing frameworks. Build on top of the new generation of cognitive radios, called CIRN, we designed and implemented a system that enables spectrum sharing with the incumbents and provides a high level of protection while maximizing channel utilization in the available spectrum, time and frequency, when the incumbent does not use it. Compared to new approaches like CBRS and LSA, our system does not require any central infrastructure to control and grant access to the shared spectrum. It only requires that the incumbents collaborate via sharing information on their status, i.e., if other users are causing interference above given security level.

We proposed and implemented an AI-based two-step algorithm that protects the incumbent. This algorithm uses spectrum data and information provided by the incumbent to recognize, learn, and proactively predict the transmission pattern of the incumbent in near real-time, the algorithm takes $300 \mathrm{~ms}$ to obtain a prediction, with high accuracy, above $95 \%$ of the predictions were correct. We have experimentally validated our architecture using Colosseum, the world's largest $\mathrm{RF}$ channel emulator built for the SC2 challenge, showed that CIRNs could collaborate, share spectrum, and protect the incumbents autonomously.

Our future work focuses on two directions. First, in the design and evaluation of mechanisms to incentivize, reward, and penalize the CIRN in 5G deployments to guarantee the protection of the incumbents for CBRS and LSA deployments. Second, how collaboration impacts the performance of the CIRN by analyzing and evaluating the trade-off between sharing additional information, e.g., GPS location or spectrum usage, and maximizing the use of local knowledge to predict the behavior of the others CIRNs.

\section{ACKNOWLEDGMENT}

The authors would like to thank DARPA to organise the SC2 competition and provide access to the Colosseum testbed.

\section{REFERENCES}

[1] Vodafone, "An industrial 5g spectrum policy for europe vodafone public policy paper." [Online]. Available: https://www.vodafone.com/content/dam/vodcom/files/public-policy/ 5g-report/an-industrial-5g-spectrum-policy-for-europe.pdf (Visited on 06-January-2020).

[2] GSMA, "5g spectrum. gsma public policy position." [Online]. Available: https://www.gsma.com/spectrum/wp-content/uploads/2019/ 09/5G-Spectrum-Positions.pdf (Visited on 06-January-2020).

[3] M. Matinmikko, H. Okkonen, M. Palola, S. Yrjola, P. Ahokangas, and M. Mustonen, "Spectrum sharing using licensed shared access: the concept and its workflow for lte-advanced networks," IEEE Wireless Communications, vol. 21, no. 2, pp. 72-79, April 2014.

[4] P. Marshall, Three-Tier Shared Spectrum, Shared Infrastructure, and a Path to 5G. Cambridge University Press, 2017.

[5] M. M. Sohul, M. Yao, T. Yang, and J. H. Reed, "Spectrum access system for the citizen broadband radio service," IEEE Communications Magazine, vol. 53, no. 7, pp. 18-25, July 2015.

[6] M. Mustonen, M. Matinmikko, M. Palola, S. Yrjölä, and K. Horneman, "An evolution toward cognitive cellular systems: licensed shared access for network optimization," IEEE Communications Magazine, vol. 53, no. 5, pp. 68-74, May 2015.

[7] DARPA, "Darpa spectrum collaboration challenge." [Online]. Available: https://www.spectrumcollaborationchallenge.com/ (Visited on 06-January-2020).

[8] P. Tilghman, "Will rule the airwaves: A darpa grand challenge seeks autonomous radios to manage the wireless spectrum," IEEE Spectrum, vol. 56, no. 6, pp. 28-33, 2019.

[9] S. D. Giannoulis, C. Donato, R. Mennes, F. A. Pereira de Figueiredo I. Jabandzic, Y. De Bock, M. Camelo, J. Struye, P. Maddala, M. Mehari, A. Shahid, D. Stojadinovic, M. Claeys, F. Mahfoudhi, W. Liu, I. Seskar, S. Latré, and I. Moerman, "Dynamic and collaborative spectrum sharing: The SCATTER approach," in 2019 IEEE International Symposium on Dynamic Spectrum Access Networks (DySPAN) (IEEE DySPAN 2019), Newark, USA, Nov. 2019.

[10] F. A. Pereira de Figueiredo, D. Stojadinovic, P. Maddala, R. Mennes, I. Jabandžić, X. Jiao, and I. Moerman, "Scatter phy: An open source physical layer for the darpa spectrum collaboration challenge," ELECTRONICS, vol. 8, no. 11, pp. 1343:1-1343:34, 2019. [Online]. Available: http://dx.doi.org/10.3390/electronics8111343

[11] M. Camelo, A. Shahid, J. Fontaine, F. A. De Figueiredo, E. De Poorter, I. Moerman, and S. Latre, "A semi-supervised learning approach towards automatic wireless technology recognition," in Proceedings of the IEEE International Symposium on Dynamic Spectrum Access Networks (DySPAN), Newark, NJ, USA, 2019, pp. 11-14.

[12] R. Mennes, M. Claeys, F. A. P. De Figueiredo, I. Jabandžić, I. Moerman, and S. Latré, "Deep learning-based spectrum prediction collision avoidance for hybrid wireless environments," jan 2019, submitted to IEEE Access.

[13] DARPA, "Phase 3 sc2 cil project." [Online]. Available: https://github.com/SpectrumCollaborationChallenge/CIL (Visited on 06January-2020).

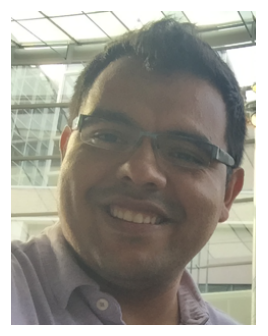

Miguel Camelo received the bachelor's degree 1203 in electronic engineering from the University of Ibague, Ibagué, Colombia, in 2006, the master's degree in systems and computer engineering from the University of Los Andes, Bogota, Colombia, in 2010, and the Ph.D. degree in computer engineering from the University of Girona, Girona, Spain, in 2014. He is currently a Researcher with the University of Antwerp-imec, Antwerp, Belgium. He has authored or coauthored several papers in journals and international conferences, and has been involved in several Spanish, Belgian, and European research projects. He also was part of the SCATTER team, a multi-disciplinary research team that participated in the DARPA SC2 competition. His research interests are in the field of applied artificial intelligence and the management of communication networks. 


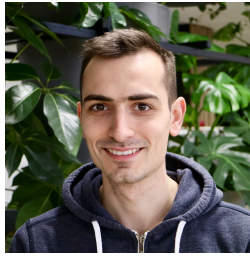

Ruben Mennes obtained a master's degree in computer science from the University of Antwerp, Belgium, in June 2016. In August 2016 he joined the Department of Mathematics and Computer Science at the University of Antwerp and imec where he is active as a Ph.D. student. The focus of his research is mainly on collaborative wireless networks and smart wireless networks, the cross-domain between wireless network technologies and machine learning. He was part of the SCATTER team where he focused on the structure, design, implementation and research of the decision-making and optimisation engine.

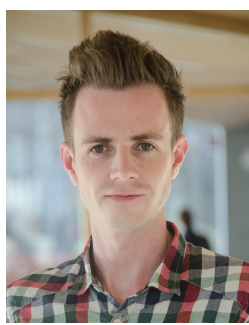

Irfan Jabandžić received his B.Sc. and M.Sc. in automatic control and electronics from Faculty of Electrical Engineering Sarajevo, Bosnia and Herzegovina, in 2012 and 2014, respectively. After working in area of embedded engineering for 2 years, in October 2016 he joined Internet Technology and Data Science Lab. at Ghent University, where he is working towards the Ph.D. degree. He was involved in DARPA Spectrum Collaboration Challenge (SC2) as MAC specialist for SCATTER team. His main research interests are in the field of wireless networks and wireless sensor networks, focusing on flexible and adaptive MAC architectures, heterogeneous wireless technologies coexistence and dynamic spectrum access.

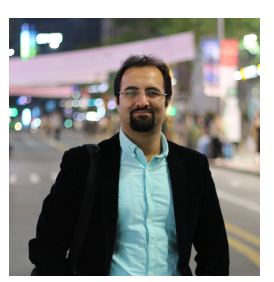

Adnan Shahid (M'15, SM'17) received the B.Eng. and the M.Eng. degrees in Computer Engineering the University of Engineering and Technology, Taxila, Pakistan in 2006 and 2010, respectively, and the Ph.D degree in Information and Communication Engineering from Sejong University, South Korea in 2015. He is currently working as a Senior Researcher at IDLab, which is a core research group of imec with research activities combined within Ghent University and the University of Antwerp. He is and has been involved in several ongoing and finished research projects: DARPA Spectrum Collaboration Challenge (SC2); European H2020 research projects such as eWINE, WiSHFUL, etc.; European Space Agency FP7 - CODYSUN, and national projects such as SAMURAI, IDEAL-IOT, Cognitive Wireless Networking Management, etc. He is a senior member of IEEE and actively involved in various research activities. He is also serving as an associate editor in various journals such as IEEE Access, Journal of Networks and Computer Application (JNCA). He is the author or co-author of more than 50 plus publications in well-known journals and conferences. His research interests include next-generation wireless communication and networks with the prime focus on resource management, interference management, cross-layer optimization, self-organizing networks, small cell networks, machine learning, 5G and $6 \mathrm{G}$ networks, etc.

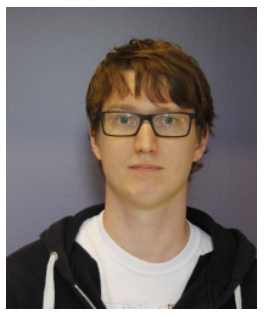

Jakob Struye received his master's degree in Computer Science at the University of Antwerp, Belgium in 2017. He then joined the Department of Computer Science at the University of Antwerp, in pursuit of a Ph.D. Initial research efforts focused on time series prediction through neural networks and other brain-like systems. He later joined the SCATTER team, building a collaborative wireless radio participating in the DARPA SC2 competition. Current research interests have shifted towards the wireless spectrum, with a focus on high-throughput low-latency mmWave communication.

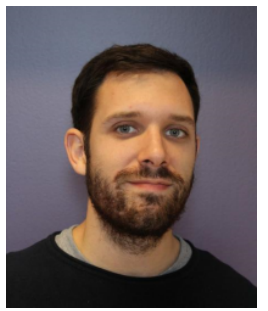

Carlos Donato received his B.Sc in Telecommunications Engineering (2013), M.Sc in Telematics Engineering (2015) and Ph.D in Telematics Engineering (2018) from Universidad Carlos III de Madrid, Department of Telematics Engineering. Currently, he holds a post-doc position at imec with IDLab research group in Antwerp. His research interests lie in wireless communications, especially LTE and IEEE 802.11 technologies, resources on demand, energy efficiency, design of network protocols, Software Defined Networking, Cloud-RAN and Artificial Intelligence techniques applied to wireless communications.

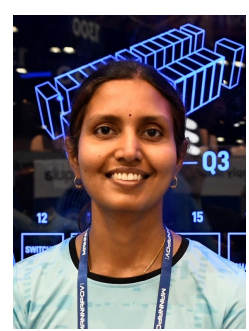

Prasanthi Maddala is a research engineer at WINLAB, Rutgers University. She obtained her Masters degree in Telecommunications from Indian Institute of Science, Bangalore, in 2006. She joined WINLAB in 2009, and worked on various projects involving SDR platforms, with her main focus on FPGA and embedded development. Her recent technical interests include SDR development for mmWave and wideband communication systems.

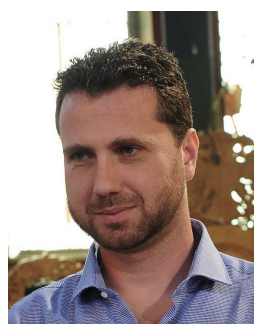

Spilios Giannoulis received his Diploma in Electrical and Computer Engineering (2001) and his PhD (2010) in the area of routing for wireless mobile sensor networks. From 2001 until 2015 he has been a researcher involved in several E.U. and national R\&D projects at Industrial systems Institute and the Applied Electronics Laboratory (APEL), University of Patras, Greece. From 2005 until 2015, he was also lecturing at the Technological Educational Institute of Patras, Greece. He is currently a postdoc researcher in imec, Belgium involved in several EU projects in the area of reconfigurable and adaptive wireless networks. $\mathrm{He}$ has served as technical leader of the $\mathrm{H} 2020$ project WiSHFUL as well as for the Scatter Team that took part in the DARPA Second Spectrum Collaboration Challenge, resulting in winning 1.5 million US dollars prizes in total from DARPA. He is currently involved in the European Space Agency CODYSUN project. His is a member of the IEEE and his main research interests are in the fields of wireless networks, mobile ad-hoc networks, wireless sensor networks, focusing on flexible and adaptive MAC and routing protocols, QoS provisioning, cross-layer and power aware system architecture design, spectrum resource management for public and licensed bands dynamic spectrum access, $4 \mathrm{G}-5 \mathrm{G}$ networks with more than 40 relevant publications in related international conferences and journals.

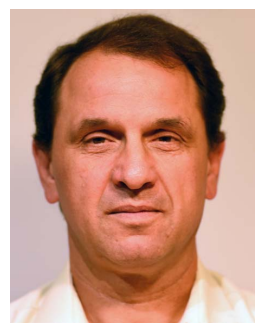

Ivan Seskar is the Chief Technologist at WINLAB, Rutgers University responsible for experimental systems and prototyping projects. He is currently the program director for the COSMOS project responsible for the New York City NSF PAWR deployment, the PI for the NSF GENI Wireless project, which resulted in campus deployments of LTE/WiMAX base stations at several US universities, and the PI for the NSF CloudLab deployment at Rutgers. He has also been the co-PI and project manager for all three phases of the NSF-supported ORBIT mid-scale testbed project at WINLAB, successfully leading technology development and operations since the testbed was released as a community resource in 2005 and for which the team received the 2008 NSF Alexander Schwarzkopf Prize for Technological Innovation. Ivan is a co-chair of the IEEE Future Networks Testbed Working Group, a Senior Member of the IEEE, a member of ACM and the co-founder and CTO of Upside Wireless Inc. 


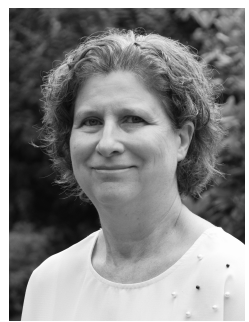

Ingrid Moerman received her degree in Electrical Engineering (1987) and the Ph.D. degree (1992) from the Ghent University, where she became a part-time professor in 2000 . She is a staff member at IDLab, a core research group of imec with research activities embedded in Ghent University and University of Antwerp. Ingrid Moerman is Program Manager of the 'Deterministic Wireless Networks' track at imec. Ingrid Moerman is also coordinating the research activities on mobile and wireless networking at Ghent University, where she is leading a research team of more than 30 members. Her main research interests include: collaborative and cooperative networks, intelligent cognitive radio networks, real-time software defined radio, flexible hardware/software architectures for radio/network control and management, Internet of Things, Next generation wireless networks $(5 \mathrm{G} / 6 \mathrm{G} / \ldots)$, and experimentally-supported research. Ingrid Moerman has a longstanding experience in running and coordinating national and EU research funded projects. At the European level, Ingrid Moerman is in particular very active in FP7/H2020 programs, where she has coordinated and is coordinating several projects (CREW, WiSHFUL, eWINE, ORCA). Ingrid Moerman was leading team SCATTER, consisting of researchers from IMEC-IDLab and Rutgers University, in the DARPA Spectrum Collaboration Challenge (SC2). The SCATTER team has been awarded with two prizes of 750000 USD each in Phase 1 and Phase 2 of the DARPA SC2 competition, and was one of the 10 finalist at the DARPA SC2 championship event organized at Mobile World Congress in LA (October 2019). Ingrid Moerman is author or co-author of more than 750 publications in international journals or conference proceedings.

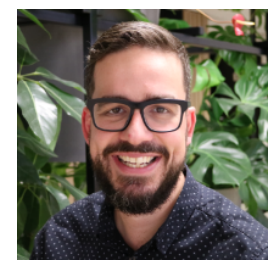

Steven Latré received the M.Sc. degree in computer science and the Ph.D. degree in computer science engineering (on autonomic network management) from Ghent University, Ghent, Belgium, in 2006 and 2011 , respectively. He is leading the IDLab Research Group, University of Antwerp-imec, Antwerp, Belgium, consisting of 100 Researchers, where he is Professor and the Director. He has authored or coauthored over 100 papers published in international journals or in the proceedings of international conferences. His personal research expertise focuses on machine learning for low power environments. Dr. Latré is a recipient of the IEEE COMSOC Award for Best the Ph.D. in Network and Service Management in 2012, the IEEE NOMS Young Professional Award in 2014, the IEEE COMSOC Young Professional Award in 2015, and the Laureate of the Belgian Academy in 2019. He is a member of the Young Academy of Belgium. 